\title{
A Book Review of Language and Meaning
}

\author{
Yin Bochun \\ School of Foreign Languages, Hunan University of Technology and Business, Hunan, China \\ Foreign Studies College, Hunan Normal University, Hunan, China \\ Email: yinbochum@163.com
}

\begin{abstract}
Language and Meaning, written by Betty Birner, specifically pays more attention on the relationships between literal and non-literal meanings, linguistic form and meaning, language and thought based on philosophical, semantic, pragmatic approaches. This book is of many significant merits and has something to be improved. In all, it is a tasteful and instructive book, and everyone to study or studying meaning should follow this book and everyone will get so many things from this book.
\end{abstract}

Keywords: Language and Meaning, book review, Betty Birner

Language is a vehicle of meaning, hence we cannot ignore meaning when language is talked about. The previous studies on language and meaning are good but lack of a macro-scope. The appearance of Language and Meaning is therefore timely and has improved this.

Language and Meaning, written by Betty Birner who is a professor in the Department of English at Northern Illinois University, USA, first published in 2018 by Routledge which is an imprint of the Taylor \& Francis Group, its LCCN is 2017036304 and ISBN is 9781138218239 . This book is one of Routledge Guides to Linguistics, a set of concise linguistic guidebooks which provide an overview of the fundamental principles of linguistic area in a jargon-free and undaunting format.

This book answers some philosophical and linguistic questions of what it means to mean. Besides this book specifically pays more attention on the relationships between literal and non-literal meanings, linguistic form and meaning, language and thought, and this book chooses philosophical, semantic, pragmatic approaches to solve these problems. This book consists of five chapters: it starts with an introduction that firstly gives a brief definition of the term "meaning", then this book defines the fields to study: meaning of words and sentences, not the life or anything else; it also states the meaning of a meaning and the role of context to meaning from semantic and pragmatic perspective, at last it discusses the relation between form and meaning of a language. Chapter 2 gives an introduction of the studies already existing on meaning from the perspective of philosophy and it focuses on these fields that language and thought, the place meaning resides, sense and reference, meaning and truth and so on. Chapter 3 analyzes the semantic structures of language, such as showing the semantic elements represented by words, and discusses the relationship between words and sentences, then shows how the meaning can be represented and how simple meanings are built up into more complex meanings. Chapter 4 states meaning from the perspective of pragmatics. This chapter discusses the relationship between what hearers understand and what speakers really want to express, namely it studies how speakers use the linguistic form encoded with literal meanings to represent expanded meanings, how hearers infer differently from what speakers really want to express with the help of the theories of pragmatics. Chapter 5 summarizes the previous chapters.

What this book characterizes are more than popular and easy to understand, besides it is also of these merits below:

1. The structure is rich and logic.

This book provides a clear and logic structure on studying meaning that it starts with an introduction on meaning and its studies related; then it brings in many great fruits on meaning respectively from the philosophical, semantic and pragmatic perspective. Philosophical perspective is anterior to semantic and pragmatic perspective because philosophy is the foundation of all subjects including semantics and pragmatics; pragmatic perspective follows semantic perspective because semantics is from linguistic 
internal system while pragmatics is from linguistic external system and the logic sequence here is inside to outside.

2. The linguistic examples are very rich

The linguistic examples the book providing are very rich in order to make the analysis for linguistic data convincing and sufficient. For example the introduction and analysis of the term "entailment" are laid with rich linguistic examples (p.62). Providing rich linguistic examples is a foundation for doing good introduction and analysis and this book do it nearly perfectly.

Nothing in the world can be perfect, and this book still has something to be improved.

This book focus on the studies on isolated dictionary meaning, but lacks of a whole network or a organizational view of a meaning. For example, we get the meaning of kick and bucket but we may not get the meaning of kick the bucket. As far as we can see, the foundation to obtain the meaning of kick the bucket is the knowledge networks of the literal meaning of kick the bucket, not the isolated literal meaning meaning of kick the bucket. We recommend that this book should pay more attention on the theory of LCCM (lexical concepts and cognitive models), put forward by Evans (2006), which can be a more systemic and efficient perspective to study meaning. Lexical Concept is a conventional meaning associated with a word/lexical item (including simple words, derivatives and compounds). Actually, word here is just the most typical example, so Lexical Concept can also include a meaning associated with a phrase or a sentence. Evans (2006) put forward further the theory of Lexical Concept and Cognitive Model, or LCCM theory for short. LCCM theory consists of an account of lexical representation and an account of meaning-construction (composition) of lexical concepts in a way which activates,or, in my terms, provides an access route through the cognitive models accessed by a given lexical concept. In short, LCCM provide a bigger and more organizational knowledge structure for a meaning. The above example Kick the bucket is illustrated from the perspective of LCCM: Kick the bucket represents originally concept KICK THE BUCKET, and the cognitive models accessed by the concept KICK THE BUCKET are essentially many knowledge networks. One of these knowledge networks is result of motion DIE (motion and patient are KICK and THE BUCKET), then we get the meaning DIE of kick the bucket based on the knowledge network and we also get the meaning construction way: metonymy (motion+patient stands for result of motion). In all, we recommend that this book should pay more attention on the theory of LCCM and add this theory to itself.

Besides the originality of this book is not strong for a major ideas or conclusions of this book does not originate from itself but from other scholars' fruits. Hence we recommend that this book should not only do the good job of theory introduction but also be more creative and put forward some ideas of the study on meaning.

In all, it is a tasteful and instructive book, and everyone to study or studying meaning should follow this book and we firmly believe that everyone will get so many things from this book.

Acknowledgements. This paper is a partial achievement of Hunan Province Social Science Funds Project "A Study on the Direct and Indirect Formal Representation Continuum of Concepts" (18YBQ074).

\section{References}

1. Evans V. (2006) "Lexical concepts, cognitive models and meaning construction," Cognitive Linguistics, pp. 491 534. 